Meta

Journal des traducteurs

Translators' Journal

\title{
La planification à long terme dans l'entreprise
}

\section{Robert Dubuc}

Volume 19, numéro 4, décembre 1974

URI : https://id.erudit.org/iderudit/002648ar

DOI : https://doi.org/10.7202/002648ar

Aller au sommaire du numéro

Éditeur(s)

Les Presses de l'Université de Montréal

ISSN

0026-0452 (imprimé)

1492-1421 (numérique)

Découvrir la revue

Citer cet article

Dubuc, R. (1974). La planification à long terme dans l'entreprise. Meta, 19(4), 208-215. https://doi.org/10.7202/002648ar d'utilisation que vous pouvez consulter en ligne.

https://apropos.erudit.org/fr/usagers/politique-dutilisation/ 


\section{LA PLANIFICATION À LONG TERME DANS L'ENTREPRISE *}

\section{Petit inventaire terminologique}

La fonction de PLANIFICATION décrit « une méthode formelle d'utilisation rationnelle des ressources matérielles et humaines de l'entreprise, en vue de maximaliser la rentabilité du capital investi et de lui assurer un développement harmonieux ${ }^{1}$ ». Ainsi envisagée, elle apparaît comme une fonction de base de la gestion, qu'il n'est pas besoin de distinguer, par une appellation distincte, de la planification nationale. La distinction qu'on établit généralement entre PLANNING et PLANIFICATION ne se justifie guère puisque les deux mots désignent essentiellement une même activité ; seule l'échelle d'application varie.

La planification peut se faire À COURT TERME, pour des besoins immédiats, ou À LONG TERME, lorsqu'il s'agit de l'orientation générale de l'entreprise. Même si les notions de court et de long termes sont très relatives, on s'entend généralement pour fixer à cinq ans et plus la durée des plans à long terme. Envisagée sous l'angle des décisions à prendre, la planification peut être CENTRALISÉE lorsque toutes les décisions doivent être prises par une autorité centrale; elle est dite DÉCENTRALISÉE si les décisions se prennent par secteur d'activité ou par unité de travail. Dans les deux cas, il doit y avoir des responsables de la planification, qu'on appelle collectivement les PLANIFICATEURS. Ces planificateurs peuvent être réunis dans un service d'état-major pour former un COMITÉ DE PLANIFICATION, chargé d'organiser la planification à l'échelle de l'entreprise. On peut aussi avoir recours à des PLANIFICATEURS CONSEILS pour résoudre certains problèmes particulièrement épineux.

L'établissement d'une planification à long terme se fait selon un PROCESSUS en quatre temps. D'abord DÉFINITION DES OBJECTIFS GÉNÉRAUX ${ }^{2}$ ou

* La terminologie anglaise de cet article est tirée de Scott, W. Brian, Long-range planning in Handbook of Business Administration, New York, McGraw-Hill, 1967, p. 3-48 à 3-59. Les équivalents français ont été mis au point par une équipe de la Banque de terminologie de l'Université de Montréal, sous la direction de l'auteur.

1. Doulé, Christian, La planification à long terme dans l'entreprise, in Entreprise, Paris, Ed. Entreprise, $\mathrm{n}^{\circ} 865$, p. 63 . Cet article fournit la terminologie de base française utilisée ici.

2. La distinction entre objective, goal et target se fonde sur l'étude suivante : En collaboration, Glossary of Corporate Planning Terms - Glossaire de la planification générale, Montréal, Hydro-Québec, 1971, p. 11. 
FONDAMENTAUX de l'entreprise ; ÉVALUATION DES RESSOURCES pour relever les forces et les faiblesses ; ÉVALUATION DE L'ENVIRONNEMENT économique, technologique et concurrentiel ; ÉTABLISSEMENT DES HYPOTHÈSES qui fonderont l'élaboration des plans. La définition des objectifs généraux se fait en fonction du RÔLE FONDAMENTAL ou de la VOCATION - comme on dit aujourd'hui - de l'entreprise. Quant à l'évaluation des ressources, elle peut se faire selon une double démarche : une démarche dite FONCTIONNELLE, qui consiste à faire un diagnostic détaillé de l'entreprise selon ses fonctions, et une DÉMARCHE GLOBALE, qui cherche à considérer l'entreprise dans son ensemble par rapport à son rôle fondamental plutôt qu'en tenant compte de ses diverses fonctions. L'évaluation de l'environnement repose, en général, sur des données statistiques d'où émanent les PRÉVISIONS ET LES EXTRAPOLATIONS touchant l'évolution probable du contexte socio-économique dans lequel l'entreprise doit croître et se développer. Une fois ces données en main, il sera possible d'établir les hypothèses qui offriront au planificateur diverses OPTIONS entre lesquelles il devra choisir.

Cette fonction de choix est à la base de l'ÉLABORATION DES PLANS. Dès qu'il est question de plans la notion de temps se change en celle de DÉLAI ${ }^{3}$. Or il y a quatre délais à envisager au stade de l'élaboration des plans : DÉLAI DE PRÉPARATION, nécessaire aux études et analyses qui vont servir de base aux plans ; DÉLAI D'EXÉCUTION, soit la durée de vie du plan; DÉLAI DE. RÉPERCUSSION couvrant non plus seulement le déroulement effectif du plan, mais le temps où s'exerce l'influence directe de l'activité planifiée (ainsi pour la planification de la fabrication d'une voiture, le délai de répercussion s'étend à la durée de vie de la voiture elle-même) ; DÉLAI PROSPECTIF qui correspond à la période où vont jouer les facteurs susceptibles d'influer sur l'évolution de l'environnement de l'entreprise. Une fois ces différents délais pris en considération, il s'agit maintenant de traduire en termes plus précis les objectifs généraux de l'entreprise de façon à déliminer l'ampleur de la planification. Il s'agit en fait d'établir la STRATÉGIE, c'est-à-dire fixer les objectifs INTERMÉDIAIRES, déterminer les ressources à mettre en œuvre. Les objectifs intermédiaires se distinguent des objectifs généraux en ce qu'ils sont QUANTIFIÉS, soit selon la MÉTHODE OPTIMALE, qui consiste à établir une norme si parfaite qu'il est en pratique impossible de l'atteindre, soit selon la MÉTHODE PRATIQUE, qui détermine, selon des techniques plus ou moins empiriques, une sorte de norme moyenne facilement accessible, soit enfin selon la METHODE PONDÉRÉE, moyen terme entre les deux précédentes, qui cherche à fixer les objectifs selon un certain nombre de variables permettant de déterminer le rendement maximal qu'on peut espérer ateindre, sans décourager les intéressés par des exigences excessives.

L'établissement de la stratégie appelle ensuite la mise au point de PROGRAMMES précis par activité. C'est l'étape TACTIQUE. Ici les objectifs deviennent IMMÉDIATS : ils doivent être taillés sur mesure par DOMAINE D'APPLICATION. On cite le plus souvent, à titre d'exemples, les PROGRAMMES DE PRODUIT, qui visent soit les GAMMES ou les FAMILLES de produits, les PRO-

3. Jean Chevalier, Organisation, Paris, Eyrolles, 1966, vol. 2, p. 113. 
GRAMMES DE CROISSANCE, par absorption ou intégration, les PROGRAMMES DE LOGISTIQUE, régissant l'utilisation rationnelle des installations.

Les plans et les programmes qui en dérivent sont maintenant en place ; il s'agit alors de veiller à leur RÉALISATION ou MISE EN EEUVRE. Cette fonction se présente comme un processus de contrôle qui permet de s'assurer que les objectifs sont atteints dans les délais impartis. C'est le CONTRÔLE DE LA QUALITÉ DU PLAN. Ce contrôle suppose que le plan a suffisamment de SOUPLESSE pour permettre les rajustements exigés par l'analyse des causes d'ÉCARTS.

Voilà pour l'essentiel, la terminologie de la planification à long terme exposée « en situation ». Nous allons maintenant reprendre ces notions sous la forme d'un petit lexique bilingue.

\section{ROBERT DUBUC}

\section{LEXIQUE FRANCAIS-ANGLAIS}

absorption

acquisition activities

Action par laquelle une entreprise fait l'acquisition d'entreprises moins importantes en conservant dans chacune les éléments qui lui sont utiles.

agent de planification

v. planificateur

ampleur

scope

Portée d'un plan.

champ d'activité

line of action

Orientation concrète prise par une entreprise dans un domaine déterminé.

comité de planification

planning group

Groupe de personnes chargées d'organiser la planification pour un secteur donné.

conseiller en planification

v. planificateur-conseil

contrôle de la qualité du plan

planning quality control

Ensemble de dispositions visant à vérifier que les plans donnent les résultats attendus.

coût d'option

opportunity cost; alternative cost

Évaluation des frais qu'entraînerait l'adoption de nouvelles méthodes de travail, de nouveaux matériaux ou d'une orientation nouvelle.

croissance

expansion; growth

Développement de l'entreprise soit sur le plan géographique, soit sur le plan financier.

délai

time measurement

Temps accordé soit pour les étapes d'élaboration du plan, soit pour la réalisation du plan.

délai de préparation

plan preparation time

Temps nécessaire à la préparation du plan. 
délai d'exécution

lead-time

Temps prévu pour la mise en œuvre de l'activité planifiée.

délai prospectif

epochal time

Période au cours de laquelle on cherche à déceler l'existence de facteurs susceptibles d'influer sur l'évolution de l'environnement de l'entreprise.

délai de répercussion

direct-impact time

Durée de vie ou d'influence directe de l'objet de la planification.

développement des ventes

market development

Organisation des ventes des nouveaux produits sur les marchés existants, création de nouveaux marchés pour les produits anciens et nouveaux.

dirigeant

executive (n.)

Personne qui prend une part importante à la direction de l'entreprise.

domaine

subject area ; subject matter

Activité qui fait l'objet de la planification.

écart

gap

Marge qui sépare prévisions et réalisations.

entreprise

company

Pris au sens générique, tout établissement industriel ou commercial exploité dans un but lucratif.

entreprise, de $l^{2}$

corporate (adj.)

Locution qui se dit de ce qui s'applique à l'ensemble de l'entreprise.

environnement

- environmental factors

Ensemble des facteurs sociaux, économiques, technologiques qui constituent le milieu dans lequel l'entreprise évolue.

étape d'élaboration

conceptual phase

Phase préliminaire conduisant à l'établissement d'un plan complet.

évaluation

appraisal

Estimation aussi rigoureuse que possible de certains facteurs et de leur interaction.

famille de produits product group

Groupes de produits apparentés par l'usage qu'on en fait ou la matière dont on les fabrique.

fixation des objectifs establishment of objectives

Action de déterminer les buts à atteindre par une activité.

fusion

amalgamation; consolidation

Procédé de concentration des entreprises dans lequel s'unissent des entreprises de même importance.

gamme de produits

product line

Produits émanant d'une même source et généralement apparentés par une caractéristique commune.

hypothèse assumption

Prévision ou extrapolation fondées sur des données précises et qui permettent de bien orienter le plan. 
installations facilities

Ensemble des outillages, dispositifs, bâtiments, etc., servant à la fabrication d'un produit ou à assurer un service.

logistique des installations facilities development

Étude des installations en fonction des personnes, des besoins présents et futurs ainsi que du rendement à obtenir.

mise en cuvre du plan implementational planning

Dernière étape de la planification proprement dite visant la réalisation des plans et programmes élaborés.

méthode fonctionnelle functional approach

Méthode d'évaluation conduisant à un diagnostic détaillé des forces et faiblesses de l'entreprise selon ses fonctions.

méthode globale

total entity approach

Méthode d'évaluation des ressources de l'entreprise envisagée dans son ensemble et en fonction de sa vocation propre.

objectif à long terme

long-term objective

Objectif dont la réalisation s'étend sur plusieurs années.

objectif fondamental

v. objectif général

objectif général basic objective ; corporate objective

But fondamental que poursuit l'entreprise par son activité.

objectif immédiat target

But visé à l'étape des programmes ou des plans tactiques.

objectif intermédiaire goal

Objectif fixé à l'étape de l'élaboration stratégique du plan.

option possible course of action ; alternative course of action

Une des orientations possibles que peut prendre un plan ou un programme.

plan à options différées

deliberate postponement plan

Plan prévoyant la possibilité de différer certaines décisions dans l'attente de renseignements supplémentaires.

planificateur planner

Spécialiste de l'élaboration des plans; responsable de l'élaboration d'un plan.

planificateur conseil consultant on planning

Spécialiste à qui l'on fait appel pour organiser la planification dans l'entreprise ou pour résoudre certains problèmes précis de planification.

planification à long terme

long-range planning; long-term planning

Mode de développement rationnel de l'entreprise prévu par des plans portant sur un avenir relativement éloigné (en moyenne cinq ans et plus).

planification centralisée

centralized planning

Forme de planification où toutes les décisions sont entre les mains d'une autorité centrale. 
planification décentralisée

decentralized planning

Forme de planification où les décisions sont prises soit par secteur d'exploitation soit par unité de travail.

planification stratégique

strategic planning

Étape intermédiaire d'édification du plan où l'on insiste surtout sur la coordination des divers éléments de façon à atteindre des objectifs intermédiaires, plus précis que les objectifs fondamentaux.

plan tactique

tactical plan

Programme précisant à court terme les objectifs immédiats, leur priorité, les délais d'exécution et les moyens à mettre en ceuvre.

politique

policy

Réponse formulée d'avance pour une catégorie de problèmes donnés.

prévisions et extrapolations statistiques statistical forecasts and projections

Études statistiques servant de base à l'élaboration des plans.

processus de planification à long terme

long range planning process

Ensemble des étapes à suivre pour établir des plans à long terme.

programme de produits

product development program

Programme recouvrant le cycle de vie des produits actuels et l'évaluation du cycle des produits à créer.

quantification des objectifs

goal setting

Précision des objectifs en fonction des quantités à produire et des délais impartis au stade de la stratégie.

quantification optimale

target approach ; carrot approach

Fixation d'objectifs par référence à une norme idéale, impossible à atteindre dans la pratique. quantification pondérée quantitative approach

Moyen terme entre la quantification optimale et la quantification pratique, cette méthode vise, en tenant compte de plusieurs variables, à fixer des objectifs qui suscitent un rendement maximal sans décourager les intéressés.

quantification pratique realistic approach

Fixation d'objectifs par référence à un rendement moyen facilement accessible et établi en général de façon empirique.

rôle fondamental

v. vocation

souplesse flexibility

Qualité d'un plan ou d'un programme qui en permet facilement l'adaptation au gré de l'évolution des circonstances.

souplesse fonctionnelle

built-in flexibility

Qualité d'un plan qui permet les adaptations nécessaires pour faire face à l'évolution des situations en prévoyant des solutions de rechange.

stratégie

strategy

Art qui consiste à dresser un plan général de campagne pour atteindre des objectifs précis. tactique (n.f.)

tactic

Art de combiner à des fins précises les moyens dont on dispose. 
variable temporelle

time variable

Données relatives aux temps et aux délais dans l'établissement d'un plan.

variable temps

v. variable temporelle

vocation mission

Champ d'activité fondamental; raison d'être de l'entreprise.

\section{LEXIQUE ANGLAIS-FRANÇAIS}

acquisition activities

alternative cost

alternative course of action

appraisal

assumption

basic objective

built-in flexibility

carrot approach

centralized planning

company

conceptual phase

consultant on planning

corporate (adj.)

corporate objective

decentralized planning

deliberate postponement plan

direct-impact time

environmental factors

epochal time

establishment of objectives

executive (n.)

expansion

facilities

facilities development

flexibility

functional approach

gap

goal

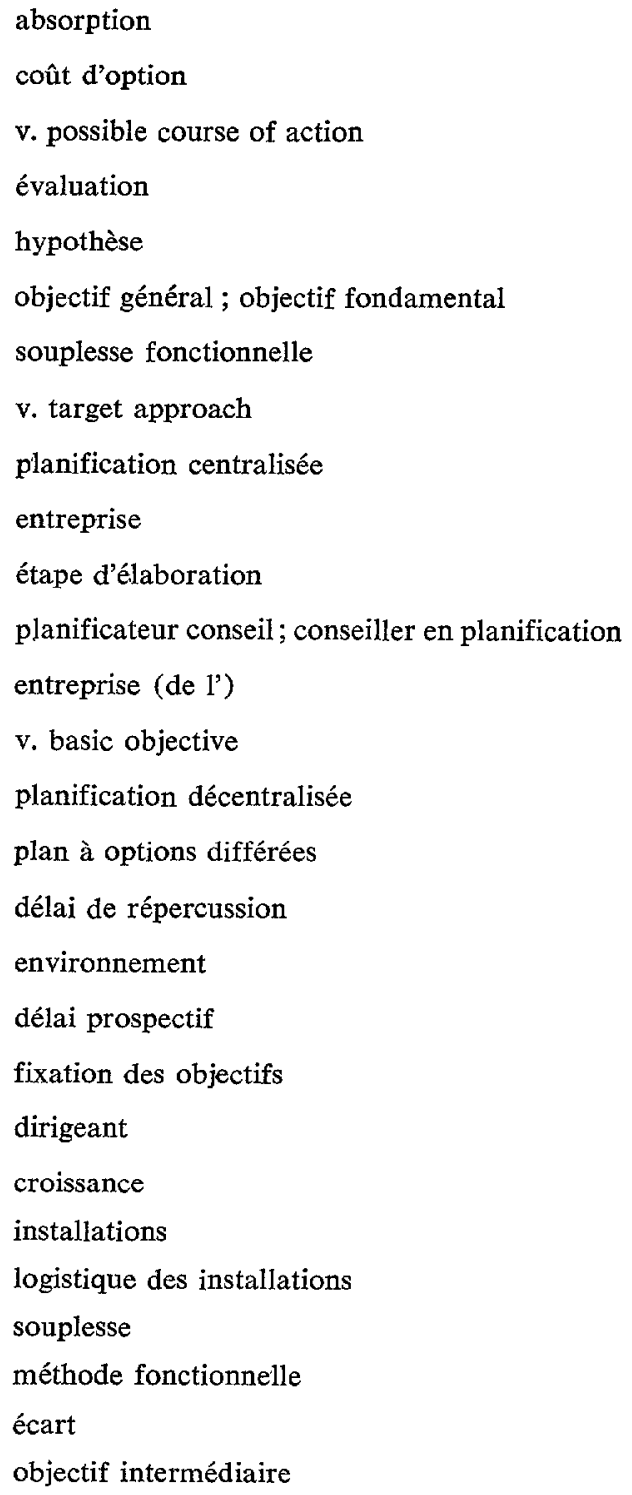


goal setting

growth

implementational planning

lead-time

line of action

long-range objective

long-range planning

long-range planning process

long-term

market development

merger

mission

opportunity cost

planner

planning group

planning quality control

plan preparation time

policy

possible course of action

product development program

product group

product line

quantitative approach

realistic approach

scope

statistical forecasts and projections

strategic planning

strategy

subject area

tactical plan

tactics

target

target approach

time measurement

time variable

total entity approach quantification des objectifs

v. expansion

mise en cuvre du plan

délai d'exécution

champ d'activité

objectif à long terme

planification à long terme

processus de planification à long terme

v. long-range

développement des ventes

absorption

vocation ; rôle fondamental

v. alternative cost

planificateur ; agent de planification

comité de planification

contrôle de la qualité du plan

délai de préparation

politique

option

programme de produits

famille de produits

gamme de produits

quantification pondérée

quantification pratique

ampleur

prévisions et extrapolations statistiques

planification stratégique

stratégie

domaine

plan tactique

tactique (n.f.)

objectif immédiat

quantification optimale

délai

variable temps ; variable temporelle

méthode globale 